\title{
Research and verification of internal and external measurement methods of large oil storage tanks capacity based on 3D laser scanning
}

\author{
Xianlei Chen ${ }^{1}$, Yexin $\mathrm{Xu}^{2}$, Yeyong Wang ${ }^{1 *}$, Zenan $\mathrm{Wu}^{1}$, Junxue Chen ${ }^{1}$, Haolei Shi ${ }^{1}$, Huadong Hao ${ }^{1}$, Zhengqian Shen ${ }^{1}$, Jianhua \\ $\mathrm{Ji}^{3}$ and Zhanfeng $\mathrm{Xu}^{3}$ \\ ${ }^{1}$ Zhoushan Institute of Calibration and Testing for Qualitative and Technical Supervision (National Metrology Testing Center for Bulk \\ Commodity Storage and Transportation Industry), Zhoushan, 316013, China \\ ${ }^{2}$ Sinochem Xingzhong Oil Staging (Zhoushan) Co., Ltd. Zhoushan, 316022, China \\ ${ }^{3}$ Endress+Hauser (China) Automation Co., Ltd. Shanghai, 200241, China
}

\begin{abstract}
Nowadays, as an important tool for petrochemical enterprises to store and transport various petrochemical products, large oil storage tanks are highly praised by a large number of oil enterprises because of their characteristics of large capacity and stable storage. For the same storage tank, on the premise of elevation determination, the accuracy of the measurement results for capacity actually mainly depends on the accurate measurement of the inner radius of each circle plate. In this paper, a $10000 \mathrm{~m}^{3}$ stroage tank T1 is selected to measure the inner radius by strapping tape method, total station internal measurement method and 3D laser scanner internal and external measurement method. Through data comparison, the accuracy of the above methods for measuring the inner radius of the first circle plate of storage tank is discussed and verified, and the difference of measurement results and accuracy between internal and external measurement of ten circle plates of storage tank by 3D laser scanner. It is concluded that the external measurement method of 3D laser scanner based on the principle of cloud to cloud splicing has poor result in tank's inner radius measurement, but it is feasible based on the principle of target ball splicing.
\end{abstract}

\section{Introduction}

For now, the capacity measurement methods of large oil storage tanks are generally used of strapping tape method, the method based on photoelectric ranging(total station method) and 3D laser scanning method[1-3]. Total station internal measurement method and 3D laser scanner internal measurement method are still the main methods of tank capacity verification. During the measurement, verification personnel are often required to get into the tank for testing. Due to the influence of residual oil and gas concentration and harmful gases, it brings hidden dangers to the safety of verification personnel. Generally, the internal measurement of large oil storage tanks can only be carried out after tank cleaning operation. Due to the high cost of tank cleaning operation, the cleaning cycle of general enterprises is about 7-8 years, which does not match the verification cycle (2 years for the first inspection and 4 years for the follow-up inspection). Therefore, a large number of oil stroage tanks need to be verified by external measurement methods.

At present, there are few external measurement tests or studies on large oil storage tanks by 3D laser scanner, and meanwhile there are few reports on the analysis of external measurement results and the feasibility of the method. As an important link in the development of oil and gas industry chain in Zhejiang Pilot Free Trade Zone, Zhoushan Islands have many large oil storage tanks, which provides a prerequisite for the corresponding tests and researches. In this paper, a $10,000 \mathrm{~m}^{3}$ storage tank T1 is selected, and the radius of the tank is measured by strapping tape method, total station internal measurement method and 3D laser scanner internal and external measurement method. Through data comparison, the accuracy of the above methods for measuring the inner radius of the first circle plate of storage tank is discussed and verified, as well as the comparison and analysis of the measurement results of the inner radius of all circle plates of the storage tank by the internal measurement and external measurement of the 3D laser scanner, so as to verify the feasibility of the external measurement method of the 3D laser scanner.

\section{Principle and limitations of existing measurement methods}

\subsection{Internal measurement method}

\subsubsection{Internal laying tape method}

*Corresponding author's e-mail: wyy0093@163.com 
Internal laying tape method is to draw the track of inner base circle at the position where the distance from the vertical weld of the first circle plate is more than $300 \mathrm{~mm}$. First, establish the starting point, and measure the circumference of the inner base circle by sticking the steel tape to the tank wall, and then re-establish the starting point at the same position to measure the circumference. Finally, take the average of the two measurement results as the circumference of the inner base circle. In the same way, the inner circumference of each circle plate is measured one by one. The limitation of this method lies in the large personal error and many influencing factors, such as obstacles in the tank and rust on the tank wall. It is dangerous and inefficient that scaffolding is required when measuring the inner circumference of other circle plates[4].

\subsubsection{Total station internal measurement method}

Total station internal measurement method is widely used to measure the inner radius of circle plates. Firstly, the instrument is set on the tripod about the geometric center of the tank bottom or floating plate, adjust to the horizontal state and input the position parameters. Secondly, set the starting direction, take the horizontal dial direction as the $\mathrm{X}$ axis of the coordinate system and the plumb line through the center of the instrument as the $\mathrm{Z}$ axis, so as to form the measurement coordinate system O-XYZ. Finally, collect the relevant coordinates of the inner wall of the oil tank every certain horizontal angle or distance, and ensure that the scanning circle is the horizontal section by adjusting the vertical angle[5].

Total station internal measurement method can automatically complete the measurement of the horizontal section of the circle plates of the entire oil storage tank. The denser the point collection is, the closer the calculated radius is to the true radius of the oil storage tank. This method has the advantages of high degree of automation and low labor intensity, but it also has its limitation that when the measurement angle is greater than $60^{\circ}$, the error will increase sharply.

\subsection{3. $3 D$ laser scanning}

The 3D laser scanner can obtain the distance D of the measured point by adopting the principle of laser ranging. The built-in precision clock control encoder is used to synchronously measure the transverse scanning angle $\alpha$ and longitudinal scanning angle $\beta$, and then the threedimensional coordinates of the measured point $(\mathrm{X}, \mathrm{Y}, \mathrm{Z})$ on the surface of the object is established (equation (1))[6].

$$
\left\{\begin{array}{l}
\mathrm{X}=\mathrm{D} \cos \beta \cos \alpha \\
\mathrm{Y}=\mathrm{D} \cos \beta \sin \alpha \\
\mathrm{Z}=\mathrm{D} \sin \beta
\end{array}\right.
$$

The 3D laser scanning method can quickly obtain the $3 \mathrm{D}$ coordinates of a large number of objects surface and form the 3D space model. The 3D laser scanner is based on the instrument site as the center to scan the spatial coordinates of the surrounding objects. Only one time scan can complete the acquisition of the $3 \mathrm{D}$ information of inner wall of the tank when the instrument is set inside the tank. The advantages of 3D laser scanning method are fast measurement speed and large scanning points. The limitation is that the latest $3 \mathrm{D}$ laser scanner ranging accuracy up to $1 \mathrm{~mm}+10 \mathrm{ppm}$, but the angle measurement accuracy is low(generally 19", the highest is $8 ")$.

\subsection{External measurement method}

\subsubsection{Strapping tape method}

The principle and measurement process of strapping tape method are the same as the internal laying tape method which is mentioned above.Because it is a direct measurement that is less affected by other factors. So the results are accurate and widely recognized at home and abroad.The limitations include high labor intensity, long measurement time and many collaborative personnel. In addition, except for the measurement of the first circle plate and the comparison of the oil storage tank capacity measurement methods, it is usually not used as the measurement method of the radius of other circle plates that scaffolding is needed.

\subsubsection{Total station external measurement method}

Total station external measurement method is to realize the measurement requirements of radial deviation method by measuring outside the oil storage tank. After the total station is set up, align the left and right sides of the oil storage tank, read the included angle, and then position the instrument to the $1 / 2$ included angle, which is the radial direction. When the total station aims at the $1 / 4$ and $3 / 4$ positions of each circle plate, the radial distance (horizontal distance) to each circle plate can be automatically measured according to the operation steps of the total station internal measurement method, and compared with the horizontal distance of the base circle, so as to obtain the radius of other circle plates. The advantage of total station external measurement method is low labor intensity, but the limitation is that it needs to strap tape of the base circle before the total station external measurement, and the number of measuring stations is large. Some bus bars may be blocked in the measurement process, which makes it impossible to set up the total station.

\section{Verification of internal measurement method for tank capacity based on 3D laser scanning}

\subsection{The measurement results and comparison of measurement methods}

\subsubsection{Measurement results of strapping tape method}

According to the calculation formula (equation (2)) of inner radius of strapping tape method and temperature 
correction formula (equation (3)), the inner radius of the first circle plate is $14989.80 \mathrm{~mm}$. Suppose that $R$ is the inner radius, $C_{1}$ is the first measured circumference at $3 / 4$ position of the circle plate, $C_{2}$ is the second measured circumference at $3 / 4$ position of the circle plate, $\Delta l$ is the sum of welds span correction values of steel tape, $\Delta l_{\text {r }}$ is the modification of the length of steel tape, $\sigma$ is the thickness of tank wall, $R_{\mathrm{i}}$ is the inner radius value after temperature correction, $\mathrm{T}$ is the average temperature of the circle plate, $\alpha_{\mathrm{r}}$ is the linear expansion coefficient of steel tape, $\alpha_{\mathrm{t}}$ is the linear expansion coefficient of $\operatorname{tank}$ (mostly be made of carbon steel, the value generally is $\left.0.000012 /{ }^{\circ} \mathrm{C}\right)$.

$$
\begin{aligned}
& R=\frac{\left(C_{1}+C_{2}\right) / 2+\Delta l+\Delta l_{\mathrm{r}}}{2 \pi}-\sigma \\
& R_{\mathrm{i}}=R \times\left[1+(\mathrm{T}-20) \quad\left(\alpha_{\mathrm{r}}-\alpha_{\mathrm{t}}\right)\right]
\end{aligned}
$$

\subsubsection{Internal measurement results of total station}

A total of 48 points are measured at $3 / 4$ of the first circle plate of the storage tank $\mathrm{T}-1$ by total station. The performance parameters of total station include angle measurement accuracy of 0.5 seconds and prism free ranging accuracy of $1 \mathrm{~mm}+1 \mathrm{ppm}$ (in the range of $0.5 \sim 100$ meters). Through calculation after removing miscellaneous points by the post-processing software of total station and temperature correction, the inner radius of total station is $14988.07 \mathrm{~mm}$.

\subsubsection{Internal measurement results of $3 D$ laser scanner}

In this test, the performance parameters of $3 \mathrm{D}$ laser scanner-1 include the shortest scanning distance of $0.6 \mathrm{~m}$, the farthest of $153.49 \mathrm{~m}$, the ranging accuracy of $\pm 2 \mathrm{~mm}$ at $10 \mathrm{~m}$ and $25 \mathrm{~m}$, and the maximum scanning rate of 97,6000 $\mathrm{pts} / \mathrm{sec}$. The performance parameters of 3D laser scanner2 include the angle accuracy of 19 arcsec for vertical/horizontal angles, the $3 \mathrm{D}$ accuracy of $2 \mathrm{~mm}$ at $10 \mathrm{~m}$ and $3.5 \mathrm{~mm}$ at $25 \mathrm{~m}$, the ranging accuracy of $1 \mathrm{~mm}$, and the maximum scanning rate of $2,000,000 \mathrm{pts} / \mathrm{sec}$. The measurement results of 3D laser scanner-1 and 3D laser scanner- 2 are processed by post-processing software. Through temperature correction, the inner radius of the first circle plate of 3D laser scanner-1 is $14988.57 \mathrm{~mm}$, and that of 3D laser scanner-2 are $14988.44 \mathrm{~mm}, 14988.45 \mathrm{~mm}$ and $14988.67 \mathrm{~mm}$ respectively.

\subsubsection{Analysis of measurement results}

(1) The inner radius of $3 / 4$ position of the first circle plate which is obtained by strapping tape method and total station internal measurement method is $14989.80 \mathrm{~mm}$ and $14988.07 \mathrm{~mm}$ respectively that the difference is $1.73 \mathrm{~mm}$ (Figure 2). It shows that the comparison result is good.

(2) The inner radius of $3 / 4$ of the first circle plate which is obtained by internal measurement method of 3D laser scanner-1 is $14988.57 \mathrm{~mm}$, and the average inner radius which is obtained by internal measurement method of 3D laser scanner-2 is $14988.52 \mathrm{~mm}$. The difference between the results is $0.05 \mathrm{~mm}$, and the difference between the inner radius which is obtained by internal measurement method of 3D laser scanners and that is obtained by stapping tape method is $1.23 \mathrm{~mm}$ and $1.28 \mathrm{~mm}$ respectively(Figure 1). The comparison results are good, which shows that the internal measurement method of 3D laser scanner has a very significant advantage in tank capacity calculation.

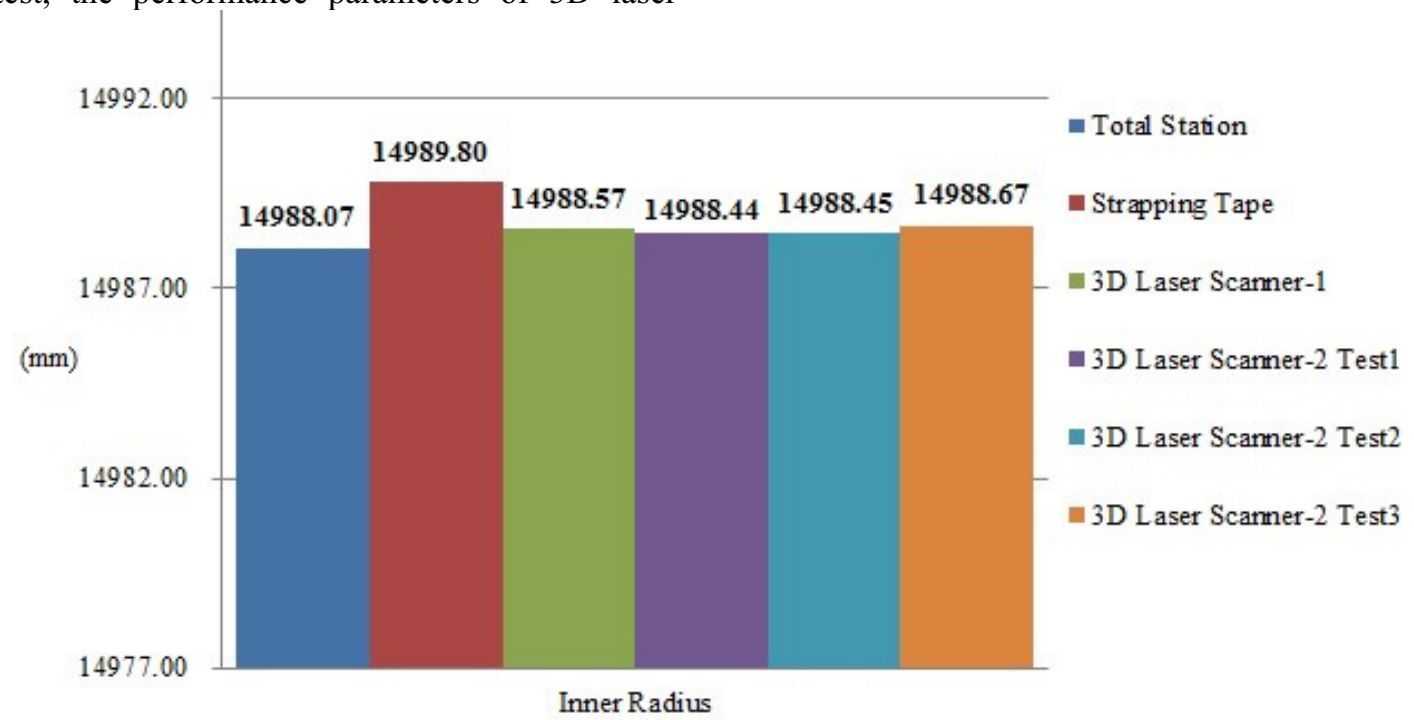

Figure 1. The results of strapping tape method and internal measurement methods.

\subsection{Repeatability test of internal measurement of 3D laser scanner-2}

A total of 3 times by 3D laser scanner-2 is used for internal measurement of oil storage tank T-1. The scanning resolution is $1 / 5$, the scanning quality is $4 \mathrm{X}$, and the scanning time is about 5 minutes. The standard deviation(STD) of repeatability test results show that the standard deviation of inner radius measurement of each circle plate is less than $1 \mathrm{~mm}$, which meets the measurement repeatability requirements (Figure 2). 


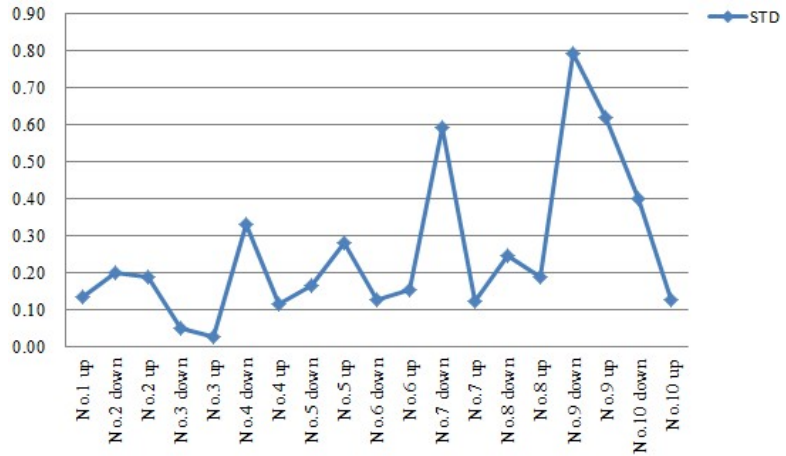

Figure 2. Repeatability test results of internal measurement of $3 \mathrm{D}$ laser scanner-2.

\section{Research and verification of external measurement method for oil storage tank capacity based on 3D laser scanning}

\subsection{The principle of external measurement}

\subsubsection{The principle of target ball splicing}

A special ball with a fixed radius $(75 \mathrm{~mm})$ is usually placed in the measurement scene when the target ball is used as a common point for splicing. When the 3D laser scanner scans from different perspectives, it can scan $1 / 3 \sim 1 / 2$ of the ball (different placement angles). The spherical surface of the target ball can be fitted by using the scanning data of the ball, and then the coordinates of the spherical center are calculated according to the relevant parameters of the special ball. The spherical center of the same name is the common point of different stations. The data of the front and rear stations can be registered by using three or more non collinear spherical centers of the same name observed from the front and rear perspectives[7].

\subsubsection{The principle of cloud to cloud splicing}

There is no need to set targets or station coordinates based on the principle of cloud to cloud splicing. The common areas of two adjacent stations are overlapped by the point cloud data processing software, and then the coordinate transformation parameters between the coordinate systems of the adjacent stations are calculated according to the iterative closest point (ICP) algorithm to complete the point cloud splicing[8].

\subsection{External measurement process}

$3 \mathrm{D}$ laser scanner-2 is used to scan oil strorage tank T-1 that based on the principle of target ball splicing and cloud to cloud splicing. Five standard target balls with a radius of $75 \mathrm{~mm}$ are used in this test. First, three target balls are placed in the first site, and then two target balls are placed in the second site, and so on(Figure 3). Eight sites are set up around the tank(L1-L8). 3D laser scanner-2 is used for scanning that the scanning resolution is $1 / 5$, the scanning quality is $4 \mathrm{X}$, and the scanning time is about 5 minutes(totally nine stations(S1-S9)).

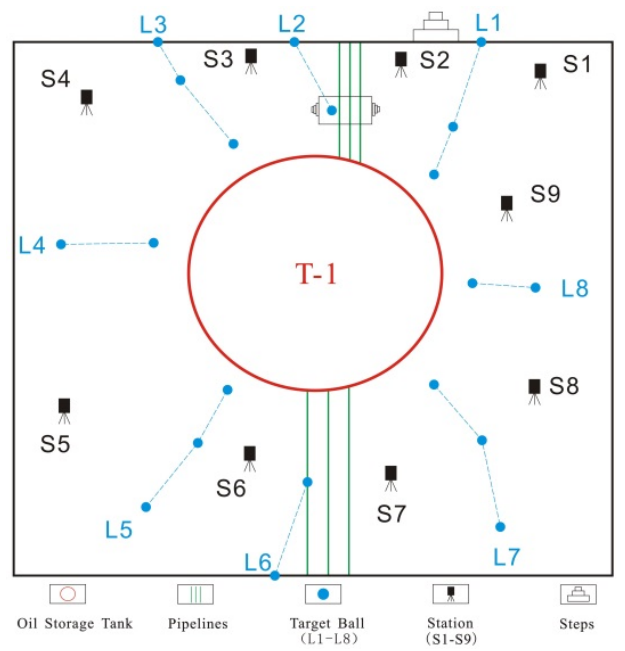

Figure 3. The figure of sites and stations layout of test area.

\subsection{Comparison of internal and external measurement results of 3D laser scanner}

As shown in Figure 4a, the comparation of the innerl radius change curve $\left(R_{b e}\right)$ which is obtained by external measurement based on the principle of target ball splicing, the inner radius change curves $\left(R_{c e}\right)$ which is obtained by external measurement based on the principle of cloud to cloud splicing and the internal radius change curves $\left(\mathrm{R}_{\mathrm{di}}\right.$, $\mathrm{R}_{\mathrm{e}-1}, \mathrm{R}_{\mathrm{e} i-2}$ and $\mathrm{R}_{\mathrm{ei}-3}$ ) which are obtained by internal measurement show that whether it is measured externally or internally, the trend of the change curve of the inner radius of the circle plates measured by the $3 \mathrm{D}$ laser scanners is almost consistent. 

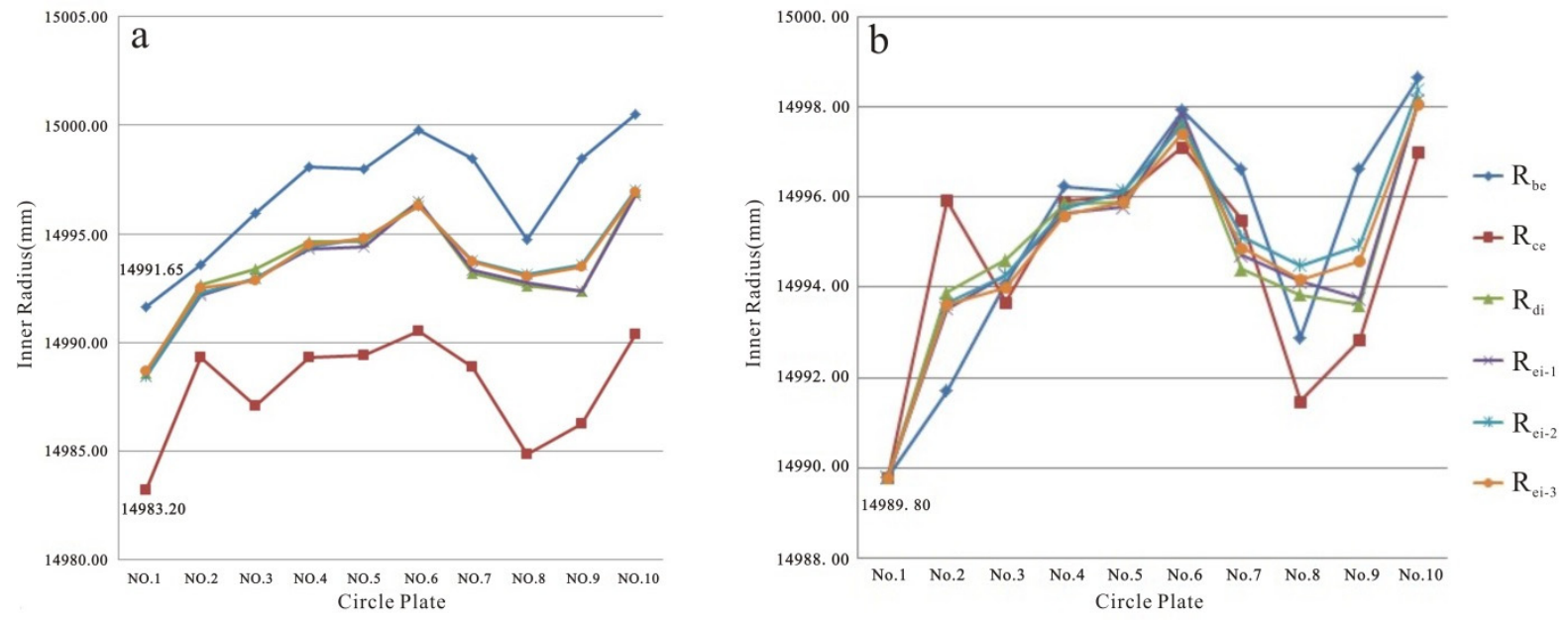

Figure 4. Comparison of internal and external measurement results of 3D laser scanner.

The inner radius of the first circle plate which is obtained by 3D laser scanner-2 external measurement method based on the principle of target ball splicing and the principle of cloud to cloud splicing is $14991.65 \mathrm{~mm}$ and $14983.20 \mathrm{~mm}$ respectively, and the difference with the result of strapping tape method is $1.85 \mathrm{~mm}$ and $6.60 \mathrm{~mm}$ respectively. The result which is obtained by the principle of target ball splicing is acceptable, but the error based on the principle of cloud to cloud splicing is large, which cannot be adopted and the comparison result is unacceptable(Figure 4a).

For each circle plate, the inner radius value $\left(\mathrm{R}_{\mathrm{be}}\right)$ based on the principle of target ball splicing is larger than that of the inner radius values of the internal measurement, and the inner radius value $\left(\mathrm{R}_{\mathrm{ce}}\right)$ based on the cloud to cloud splicing is smaller than that of the inner radius value in the internal measurement(Figure 4a). When the inner radius of the first circle of six groups of test data is unified as the inner radius which is obtained by strapping tape method based on the principle of radial deviation(Figure $4 b$ ). It clearly shows that the variation range of the inner radius curve based on the principle of the target ball splicing is smaller than that based on the principle of cloud to cloud splicing for inner radius change curves.

\section{Conclusions}

In general, this paper once again confirmed 3D laser scanner internal measurement method for large oil storage tank capacity measurement has a very significant advantage. For the first circle plate, the result based on the principle of target ball splicing is acceptable, but the result based on cloud to cloud splicing is not acceptable. For other circle plates, through the comparison of the internal and external measurement results, it is concluded that the trend of the inner radius change curves of the circle plates is almost consistent. Otherwise, there are many factors affecting the capacity measurement, but only for the influence of inner radius without considering the bottom volume of oil storage tank, the capacity deviation between the external measurement value based on the principle of target ball splicing and the average value of internal measurement of scanner is only 0.44 per thousand, but the capacity deviation between the external measurement value based on the principle of cloud to cloud splicing and the average value of the internal measurement of scanner is 0.75 per thousand. It represents that the external measurement result based on the principle of target ball splicing is acceptable, but that based on the principle of cloud to cloud splicing is unacceptable. Based on the principle of radial deviation, the capacity deviation between the external measurement value based on the principle of target ball splicing and the average value of the internal measurement of scanner is 0.027 per thousand, and the capacity deviation between the external measurement value based on the principle of cloud to cloud splicing and the average value of the internal measurement of scanner is 0.044 per thousand. It shows that the capacity deviation results of the external measurement of 3D laser scanner based on the principle of radial deviation are acceptable, but the external measurement result based on the principle of target ball splicing is better. And it is necessary to carry out further research on the influence of the number of stations, empty tanks and tanks with liquid on the splicing results of the external measurement of 3D laser scanner by adding test cases in the future.

\section{Acknowledgments}

This work is supported partly by the Scientific Research Project of Zhejiang Market Supervision and Administration Bureau (No.20190354), the Quality Technology Infrastructure Foundation of Zhejiang Market Supervision and Management System (No.20190132 \& No.20200133) and the Project of Zhoushan Science and Technology Bureau (No.2020C33170).

\section{References}

1. Knyva V, Knyva, M and Rainys J. (2013) New Approach to Calibration of Vertical Fuel Tanks[J]. ELEKTRONIKA IR ELECTROTECHNIKA., 19(8): 37-40.

2. Zhuang ZJ, Wang LG, Guan L, et al. (2018) Summary on Application of 3D Laser Scanning Technology in Capacity Measurement of Vertical 
Tank[J]. Contemporary Chemical Industry., 47(2): 403-410.

3. Hao HD, Li CJ, Liu Y, et al. (2018) Volume Measurement Method of Vertical Metal Tank Based on Laser Scanning[J]. Acta Metrologica Sinica., 39(2): 222-227.

4. JJG168-2018. (2018) Chinese Metrology Regulation: Vertical metal tank capacity[S]. Beijing: China Standard Press.

5. Tian W, Sun XP. (2020) Discussion on capacity measurement method of vertical metal $\operatorname{tank}[\mathrm{J}]$. Industrial Metrology., S1: 6-8.

6. Chen XL, Hao HD, Li CJ, et al., (2014) Study on 3D laser scanner method on volume metrology of large-scale vertical tank[J]. China Measurement \& Test., 40(2): 44-48.

7. Hong DX, Li ZC and Wang L. (2012) Point cloud splicing accuracy test based on spherical target[J]. Bulletin of Surveying and Mapping., S1: 77-79.

8. Cheng YH, Lin YL, Bai ZD, et al., (2021) Evaluation of data registration methods in terrestrial 3D laser scanning[J]. Engineering of Surveying and Mapping., 30(1): 20-29. 